\title{
Finite-particle tracking reveals submicroscopic-size changes of mitochondria during transport in mitral cell dendrites
}

\author{
Arne Gennerich $^{1,2}$ and Detlev Schild ${ }^{1}$ \\ ${ }^{1}$ Department of Neurophysiology and Cellular Biophysics, University of Göttingen, Humboldtallee 23, \\ 37073 Göttingen, Germany \\ ${ }^{2}$ Department of Cellular and Molecular Pharmacology, University of California San Francisco, \\ San Francisco, CA 94143-2200, USA \\ E-mail: gennerich@gmail.com and dschild@gwdg.de
}

Received 12 November 2005

Accepted for publication 23 January 2006

Published 16 February 2006

Online at stacks.iop.org/PhysBio/3/45

\begin{abstract}
The mechanisms of molecular motor regulation during bidirectional organelle transport are still uncertain. There is, for instance, the unsettled question of whether opposing motor proteins can be engaged in a tug-of-war. Clearly, any non-synchronous activation of the molecular motors of one cargo can principally lead to changes in the cargo's shape and size; the cargo's size and shape parameters would certainly be observables of such changes. We therefore set out to measure position, shape and size parameters of fluorescent mitochondria (during their transport) in dendrites of cultured neurons using a finite-particle tracking algorithm. Our data clearly show transport-related submicroscopic-size changes of mitochondria. The observed displacements of the mitochondrial front and rear ends are consistent with a model in which microtubule plus- and minus-end-directed motor proteins or motors of the same type but moving along anti-parallel microtubules are often out-of-phase and occasionally engaged in a tug-of-war. Mostly the leading and trailing ends of mitochondria undergo similar characteristic movements but with a substantial time delay between the displacements of both ends, a feature reminiscent of an inchworm-like motility mechanism. More generally, we demonstrate that observing the position, shape and size of actively transported finite objects such as mitochondria can yield information on organelle transport that is generally not accessible by tracking the organelles' centroid alone.
\end{abstract}

M This article features online supplementary data

\section{Introduction}

Most bidirectional long-distance mitochondria transport is thought to be achieved by the microtubule transport system $[1,2]$. Transport in the microtubule plus-end direction is driven by motor proteins of the kinesin family, whereas transport in the microtubule minus-end direction is mainly carried out by cytoplasmic dynein $[3,4,5]$. While the properties of single motor proteins have been extensively studied in vitro [6], their in vivo function and the mechanisms of coordinating opposite-polarity motors in living cells are as yet poorly understood (reviewed in [7, 8]). Several studies have suggested that opposite-polarity motors can associate with the same cargo simultaneously and that motors with the required directionality become activated while at the same time the activities of motors with opposite directionality are downregulated [9-14]. Such a mechanism must be able to rapidly coordinate the activity of opposing motors to prevent a tug-of-war [10, 15]. Bidirectional organelle transport has been found, indeed, to depend on motor cofactor proteins 
such as the protein complex dynactin (e.g. [15-19]) and the regulator proteins Klar [20] and Halo [12]. In addition, phosphorylation/dephosphorylation of motor proteins may play a role in the regulation of bidirectional organelle transport [21-23].

Despite the progress on dissecting the molecular mechanisms underlying bidirectional transport, there is as yet no direct evidence for coordination or tug-of-war between opposing motors [7]. Whereas a mechanism entirely based on competitive activity of opposing motors is unlikely and is not supported by the current state of research [8], a combination of motor coordination and tug-of-war (failed coordination) could potentially explain the oscillatory forward and backward movements of cargos during net unidirectional transport. Direct visualization of the activities of motor proteins while attached to the same cargo is presently not feasible. New biophysical means have to be employed to this end because the commonly observed center-of-mass movement of organelles generally does not allow one to gain direct evidence on the simultaneous activity of opposing motor proteins. Studying positions, shapes and sizes of actively transported finite objects such as mitochondria, on the other hand, could potentially yield direct evidence for movement-related changes in size and shape of transported targets. The simultaneous activity of opposite-polarity motors attached to the front and rear ends of the cargo, respectively, could cause detectable simultaneously opposing motor steps. Such investigations, however, have not been undertaken yet.

Here we made a first step in this direction and examined whether bidirectional transport affects the cargo's shape and size on a submicroscopic-scale level. We applied a recently introduced finite-particle tracking (FPT) algorithm [24] and studied mitochondria transport in dendrites of cultured neurons of Xenopus laevis tadpoles. Mitochondria were chosen because these organelles often have ellipsoid- or sausagelike shapes that are easy to analyze with the FPT algorithm. Our investigation reveals, for the first time, transport-induced submicroscopic-size changes of mitochondria. We show that both mitochondrial ends can undergo similar time-delayed characteristic movements. One of the cargo ends seems to dictate the direction and speed of transport while the opposite end is locked-in later and thus lags behind.

In summary, we demonstrate that the FPT analysis of actively transported finite objects such as mitochondria opens a window for studying movement-related size changes and potentially other mechanisms of bidirectional organelle transport in living cells.

\section{Materials and methods}

\section{Cell culture and staining of mitochondria}

Cultured neurons of the olfactory bulb of Xenopus laevis tadpoles were prepared and characterized as previously described [25] (see the supplementary data available from stacks.iop.org/PhysBio/3/45 for details). Living mitochondria were labeled with MitoTracker Orange (Molecular Probes, Leiden, The Netherlands) (see the supplementary data available from stacks.iop.org/PhysBio/3/45 for details).

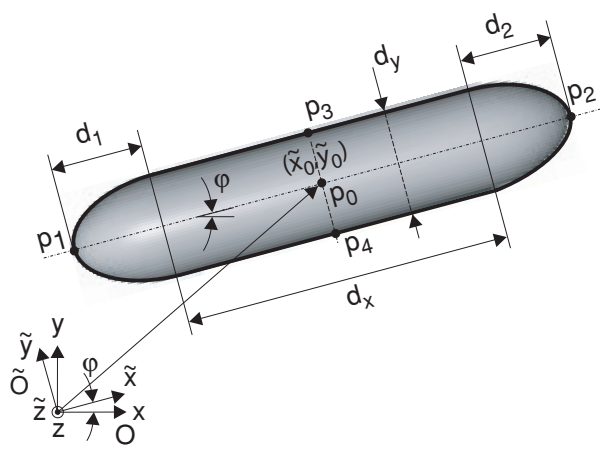

Figure 1. Schematic representation of the three-dimensional 'sausage-model'. The position of the object within an image is defined by the coordinates $\left(\tilde{x}_{0}, \tilde{y}_{0}\right)$ given in the rotated coordinate system $\tilde{O}$, where its orientation is defined by the angle $\varphi$ with respect to the $x$-axis of the coordinate system $O$. Assuming movement in the negative $\tilde{x}$-direction, the positions of the center, the front end, the rear end, the right border and the left border of the model are given by the points $p_{0}, p_{1}, p_{2}, p_{3}$ and $p_{4}$, respectively, with respect to $\tilde{O}$.

\section{Experimental setup}

Measurements were performed using a home-built instrument combining the advantages of fluorescence correlation spectroscopy (FCS) and confocal laser scanning microscopy (CLSM) as previously described [24, 26]. The minimum scanning time per line was set to $1 \mathrm{~ms}$. To allow both fast image acquisition and the observation of long-distance transport processes, the long axis of the dendrite of interest was aligned with the $x$-axis of the scanning field (corresponds to the scanning direction) by rotating the culture dish around the optical axis.

\section{Finite-particle tracking (FPT) analysis}

Position, orientation, shape and size parameters of mitochondria were determined using a recently introduced FPT algorithm [24]. FPT analysis is based on fitting a theoretical intensity distribution $I_{\mathrm{obj}}(x, y, z=0)$ of a $3 \mathrm{D}$ model object to the measured fluorescence image of the organelle. The geometry of the object is modeled by a cylinder with two hemiellipsoidal caps attached to it (figure 1, 'sausagemodel'). Here, we used an approximation of the sausage model by a stack of rectangular slices (see equations (6), (7) and (11) in [24]). The parameters of the algorithm are (figure 1) an amplitude factor $p$, the length $d_{1}$ of the left cap, the length $d_{2}$ of the right cap, the length $d_{x}$ of the cylinder, the cylinder diameter $d_{y}$, the angle $\varphi$ by which the major symmetry axis of the object is rotated around the $z$-axis (optical axis) with respect to the $x$-axis of the coordinate system $O$ (coincides with the coordinate system of the CLSM image, see figure $2(a))$, the center of the cylinder $p_{0}=\left(\tilde{x}_{0}, \tilde{y}_{0}\right)$ with respect to $\tilde{O}$ and a background constant $I_{\mathrm{B}}$. The length and width of the object are thus determined by the parameters $d=d_{1}+d_{x}+d_{2}$ and $d_{y}$, respectively. By varying the parameters $d_{1}, d_{2}, d_{x}$ and $d_{y}$ of the underlying model, the object can easily assume the shape of a sausage, ellipsoid or 
sphere. However, a precise detection of the object boundaries requires a homogeneous background intensity. Furthermore, caution is necessary when applying the algorithm to images of objects with a component of movement along the optical axis (out-of-focus movement), as a vertically tilted object would appear shorter than it actually is [24]. Hence, we have analyzed experimental examples in which both the average mitochondrial size and the mean mitochondrial intensity were constant over the time course of the measurement. These requirements are typically fulfilled when analyzing transport processes along microtubules oriented parallel and close to the cover slip, as, e.g., in thin axons and dendrites of cultured neurons.

\section{Statistical analysis based on data from two processes}

To determine whether the source for detected mitochondrial size fluctuations (quantized by the standard deviation $\sigma_{\mathrm{m}}$ of the measured mitochondrial length or width, respectively) is the noise inherent in a time-varying fluorescence signal, we performed statistical analysis on the experimental and corresponding simulated data (see the supplementary data available from stacks.iop.org/PhysBio/3/45). In the first step, we simulated a sequence of fluorescence images of a mitochondrion of the same shape and size as the experimental mitochondrion (containing fluorescence-based normal-distributed noise [24]) and being transported with identical mean velocities whereas the shape and size parameters were kept constant. The FPT analysis of the simulated mitochondrion yields the standard deviation $\sigma_{\mathrm{s}}$ of the noise-induced size fluctuations. Next, we calculated the ratio $\alpha=\sigma_{\mathrm{m}} / \sigma_{\mathrm{s}}$ and estimated the probability $P$ that the ratio $\sigma_{\mathrm{m}} / \sigma_{\mathrm{s}}$ can be equal to or larger than $\alpha$. Here, $P\left(\sigma_{\mathrm{m}} / \sigma_{\mathrm{s}}\right) \geqslant \alpha$ was estimated under the assumption that the underlying numbers of $\sigma_{\mathrm{m}}$ are independent and identical normal distributed ("null hypothesis'), i.e. that mitochondrial size fluctuations are noise induced only (see the supplementary data available from stacks.iop.org/PhysBio/3/45). We derived an equation based on the null hypothesis to precisely determine the maximum error for a given ratio $\alpha=\sigma_{\mathrm{m}} / \sigma_{\mathrm{s}}$ and the numbers $N$ and $M$ of the data values obtained from both experiment and simulation:

$$
\begin{gathered}
P\left(\frac{\sigma_{\mathrm{m}}}{\sigma_{\mathrm{s}}} \geqslant \alpha\right)<1-\frac{2^{1-N / 2}}{\Gamma(N / 2)} \int_{0}^{\sqrt{N-1} \alpha R} t^{N-1} \mathrm{e}^{-t^{2} / 2} \mathrm{~d} t \\
+\frac{2^{1-M / 2}}{\Gamma(M / 2)} \int_{0}^{\sqrt{M-1} R} t^{M-1} \mathrm{e}^{-t^{2} / 2} \mathrm{~d} t
\end{gathered}
$$

with $\Gamma$ being the gamma function. $P\left(\sigma_{m} / \sigma_{s}\right) \geqslant \alpha$ is estimated by calculating the minimum of the right-hand side of equation (1) by varying the parameter $R>1$ numerically. For further details underlying the statistical analysis and the derivation of equation (1), see the supplementary data available from stacks.iop.org/PhysBio/3/45.

\section{Zero-crossing analysis}

Zero-crossing analysis (see, for example, [27]) was used to quantitatively analyze the persistence of observed mitochondrial size changes. The FPT analysis of a hypothetical homogeneously stained fluorescent mitochondrion with constant shape and size will result in normal-distributed size changes (see supplementary figure $\mathrm{S} 1(a)$ ). The probability $p$ that noise in the images will cause an overestimated mitochondrial length (or width) in one image and an underestimated length (or width) in the next (or vice versa) is $50 \%$. The expected average number of such size transitions (herein referred to as zero crossings) for a total of $N$ images is $(N-1) p$ and the probability $P$ for measuring $N_{0}$ zero crossings is given by a binomial distribution (see the supplementary data and figure $\mathrm{S} 1(c)$ ). Using the De MoivreLaplace theorem for $(N-1) p(1-p) \gg 1$, the probability $P^{-}\left(N_{0}\right)$ for measuring a zero-crossing number smaller than or equal to $N_{0}$ is given by (supplementary data)

$$
P^{-}\left(N_{0}\right)=\frac{1}{2}\left[1-\operatorname{erf}\left(\frac{(N-1) p-N_{0}}{\sigma \sqrt{2}}\right)\right]=1-P^{+}\left(N_{0}\right),
$$

with $P^{+}\left(N_{0}\right)$ being the probability for measuring any number of zero crossings larger than or equal to $N_{0}$ and $\sigma=$ $\sqrt{(N-1) p(1-p)}$ being the standard deviation. Equation (2) was used to determine whether a measured zero-crossing number was significantly decreased or increased compared to the expected mean number of zero crossings for noise-induced size changes.

\section{Supplementary data}

Supplementary data, which provides details of calculations, statistical analysis and computer simulations, is available from stacks.iop.org/PhysBio/3/45.

\section{Results}

In order to visualize the active transport of mitochondria in living neurons and measure their shapes and sizes, we homogeneously stained mitochondria with the lipophilic cationic dye MitoTracker. To ensure non-invasive CLSM measurements, laser intensity was kept as low as possible while maintaining a sufficient signal-to-noise ratio (SNR) to allow statistically reliable FPT analysis.

Figure 2(a) shows the intensity distribution of an ellipsoidal-shaped dendritic mitochondrion along with the corresponding CLSM image (upper panel). Shown is the first of 100 sequential images. Position, orientation, shape and size of the mitochondrion in each image were obtained by FPT analysis (Materials and methods). The analysis of the first image is shown in figure $2(b)$. Depicted is the intensity distribution $I_{\mathrm{obj}}(x, y, z=0)$ of the three-dimensional model object describing the mitochondrion. An $x$-section through the experimental and theoretical intensity distribution is given in the left inset. The FPT analysis of the image sequence yields the trajectories of the five characteristic object points $p_{0}$ to $p_{4}$ as indicated in figure $2(c)$. In figure $2(d)$, we plotted the traces of the centroid and the rear and the front end of the mitochondrion, $p_{0}(\tilde{x}), p_{1}(\tilde{x})$ and $p_{2}(\tilde{x})$, respectively, with respect to the $\tilde{x}$-axis of the rotated coordinate system $\tilde{O}$, i.e., with respect to an axis parallel to the major symmetry axis of 

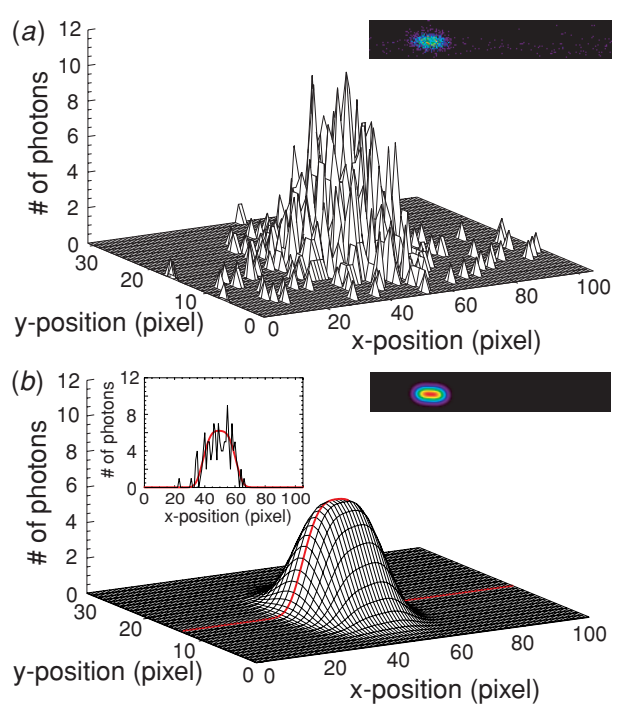

(c)
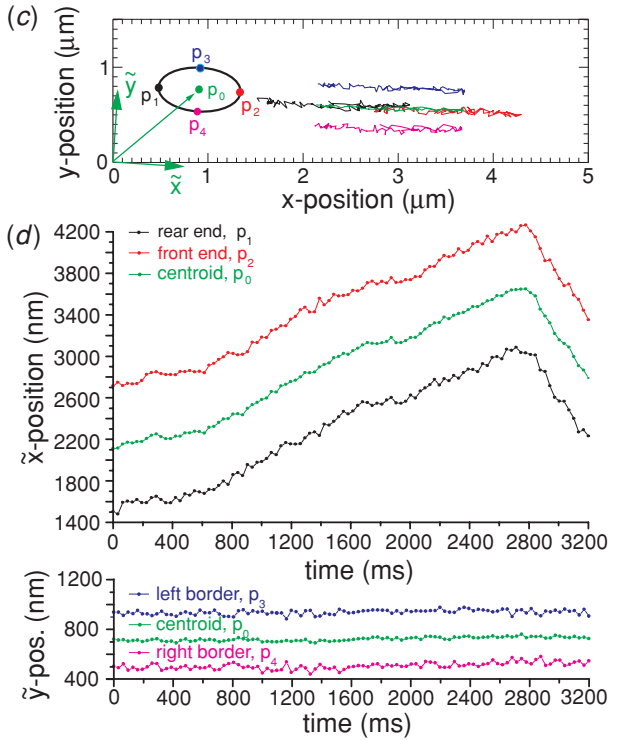

Figure 2. The FPT analysis of an actively transported dendritic mitochondrion. (a) The intensity distribution of the mitochondrion. The graph depicts the left half of the inserted CLSM micrograph (first of 100 sequential images). CLSM image size: $200 \times 32$ pixel. Image sampling interval: $32.32 \mathrm{~ms}$. Line scanning time: $1.01 \mathrm{~ms}$. Pixel dwell time: $1.25 \mu$ s. Pixel size: $44 \mathrm{~nm}$ (for parameters characterizing the object intensity, see supplementary table S1). (b) Fitted intensity distribution $I_{\mathrm{obj}}(x, y, z=0)$ of the $3 \mathrm{D}$ model object. $x$-sections along the red line through both the experimental and fitted intensity distributions are shown in the inset. Results of the fit: $p=10.853, d_{x}=0$ (fixed), $d_{1}=608 \mathrm{~nm}\left(d_{2}=d_{1}\right.$, fixed), $d_{y}=436 \mathrm{~nm}, I_{B}=0.0233, \tilde{x}_{0}=48.98$ pixel, $\tilde{y}_{0}=16.2$ pixel and $\varphi=-0.05163\left(\chi^{2}=1171.5134\right)$. The length of the ellipsoidalshaped mitochondrion is $d=1216 \mathrm{~nm}$. (c) Trajectories of the object points $p_{0}$ to $p_{4}$ (see inserted schematic). The $\tilde{x}$-axis of the rotated coordinate system $\tilde{O}$ (green) is aligned with the major symmetry axis of the mitochondrion. The angle of rotation $\varphi=$ -0.0521 (corresponds to the direction of transport) is the mean parameter obtained from the FPT analysis of the measured image sequence. $(d)$ Traces of the mitochondrial centroid $\left(p_{0}\right)$ and the front $\left(p_{2}\right)$ and rear $\left(p_{1}\right)$ ends with respect to the $\tilde{x}$-axis (upper panel). The lower panel depicts the movements of the left $\left(p_{3}\right)$ and right $\left(p_{4}\right)$ mitochondrial borders together with the centroid $\left(p_{0}\right)$ with respect to the $\tilde{y}$-axis of $\tilde{O}$. the mitochondrion (figure $2(c)$ ). The movements of the left and right mitochondrial borders, $p_{3}(\tilde{y})$ and $p_{4}(\tilde{y})$, are drawn together with the centroid $p_{0}(\tilde{y})$ with respect to the $\tilde{y}$-axis of $\tilde{O}$ (lower part of figure $2(d)$ ). The analysis reveals anterograde movement of $\sim 1.5 \mu \mathrm{m}$ over a period of $2.8 \mathrm{~s}$, followed by a rapid reversal in the retrograde direction (figure $2(d)$ ).

The centroid movement can be 'linearized' by trace sectioning and fitting straight lines to the resulting trace segments (figure 3(a)). The mitochondrion is transported in a linear fashion with various mean velocities during continuous unidirectional movement, except for a few small deviations from the fitted lines. While the traces of the mitochondrial front $\left(p_{2}\right)$ and rear $\left(p_{1}\right)$ ends reflect on average similar linear movements (figure $2(d)$ ), the deviations of the latter seem to be markedly higher than those observed for the centroid. To visualize these deviations, we superimposed the traces of the front and rear ends by subtracting the measured mean mitochondrial length from the front-end trace shown in figure $2(d)$ and then plotted the distance traveled by both ends after applying a three-point moving window (figure $3(b)$ ).

The superimposed traces reveal non-simultaneous displacements of both mitochondrial ends. To help visualize these features, we shifted the front-end trace depicted in figure $3(b)$ toward the origin of the coordinate system (figure 3(c)). Interestingly, the rear end seems to start in the lead and to dictate the movement in the anterograde direction. The front end apparently lags behind. While the rear end is displaced by $\sim 100 \mathrm{~nm}$ between the second and third images (step 1, figure 3(c)), the front end is lagging. The displacement of the rear end is then followed by an immobile period; $\sim 140 \mathrm{~ms}$ after the initial displacement of the rear end, the front end seems to catch up and makes a move over the same distance of $\sim 100 \mathrm{~nm}$ (step 2, figure $3(c)$ ). The alternating non-simultaneous mobile and immobile periods of both ends result in mitochondrial length changes as illustrated in the inset of figure 3(c). A pause of movement of both ends is then again followed by a displacement of the rear end first (step 3, figure $3(c)$ ). Following a displacement of $\sim 650 \mathrm{~nm}$ in the anterograde direction the rear end pauses for a while, and then moves forward (step 4, figure 3(c)). A similar pause is made $\sim 90 \mathrm{~ms}$ later by the front end (step 5, figure 3(c)). After a distance of $\sim 1550 \mathrm{~nm}$, the rear end seems to introduce the backward movement while the front end still moves forward resulting in simultaneously opposing displacements of both ends (step 6, figure 3(c) and gray-shaded rectangle in figure $3(b)$ ). In sum, both mitochondrial ends undergo similar characteristic movements with substantial time delay between the displacements of both ends (see arrows in figure 3(c)). To visualize the similarity of the movements of both ends, we superimposed trace segments in figure $3(b)$ and indicated the approximate time delay.

The validity of the above interpretation depends on the precision of the measurement. Noise in the images could potentially be a source for some of the observations made. Hence, we simulated the movements of a mitochondrion of the same shape and size as the experimental mitochondrion and being transported with identical mean velocities (figure 3(a)) whereas the shape and size parameters were kept 

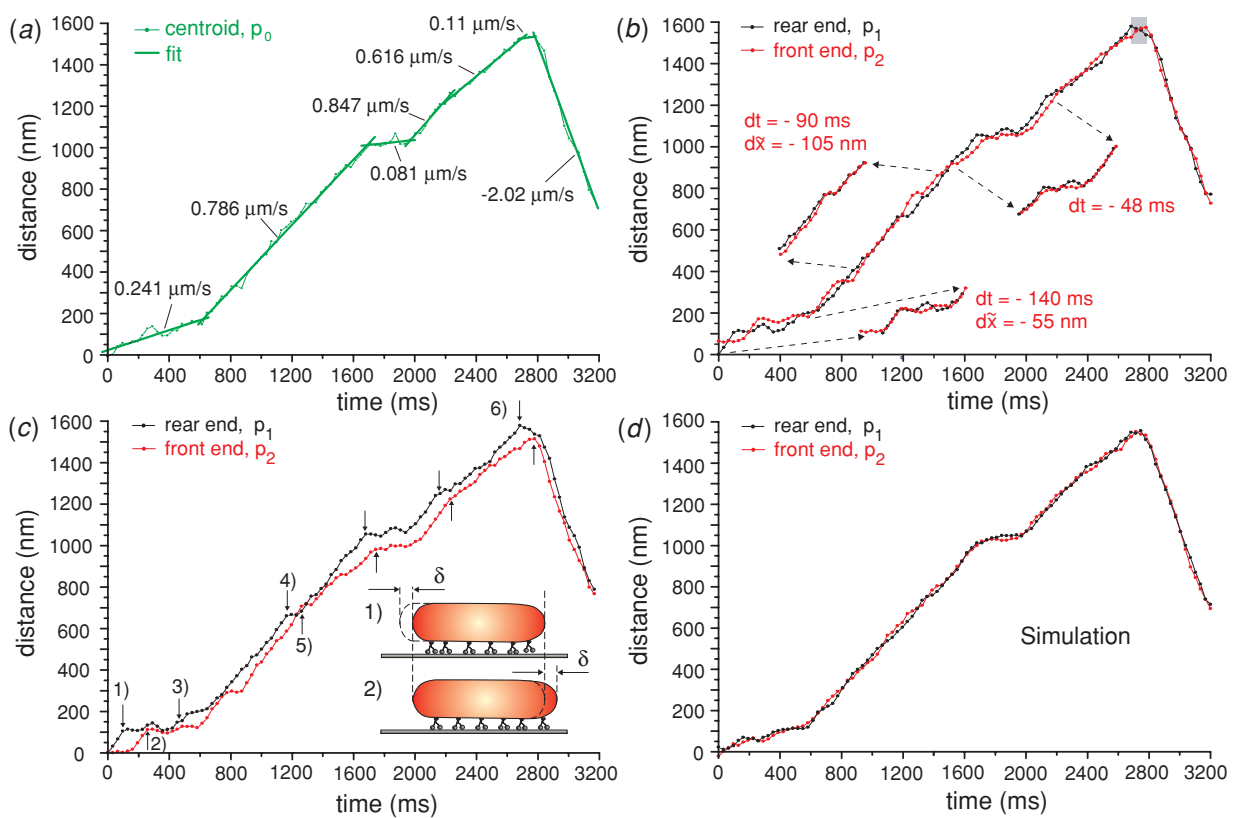

Figure 3. Displacement traces of the mitochondrial front and rear ends of experiment (see figure 2) and simulation. (a) Displacement of the centroid $\left(p_{0}\right)$ along the $\tilde{x}$-axis of $\tilde{O}$. The superimposed straight lines depict the result of the fits to the various trace sections (see the main text). (b) Superimposed smoothed displacement traces (with respect to the $\tilde{x}$-axis) of the mitochondrial front and rear ends. The superposition of the traces was achieved in two steps. First, the measured mean mitochondrial length of $\bar{d}=1178.4 \mathrm{~nm}$ was subtracted from the front end trace $\left(p_{2}\right)$ shown in the upper panel of figure $2(d)$ and second, the $\tilde{x}$-position of the rear end $\left(p_{1}\right)$ in the first image was subtracted from both the resulting trace and the trace of the rear end. Finally, we applied a three-point moving window. The inserted trace segments (see dashed arrows) were superimposed by shifting the corresponding segments of the front end trace along the time- and $\tilde{x}$-axis by the indicated values $\mathrm{d} t$ and $\mathrm{d} \tilde{x}$, respectively. The gray-shaded rectangle marks simultaneously opposing displacements of both mitochondrial ends. (c) Front and rear end traces after moving the front end trace shown in $(b)$ of this figure by $60 \mathrm{~nm}$ toward the origin of the $\tilde{x}$-axis. The inserted schematic illustrates the length changes of the mitochondrion during the first $300 \mathrm{~ms}$ of the measurement.

(d) Smoothed displacement traces of the simulated mitochondrion (a detailed description of the simulation is provided in the supplementary data and figure S2).

constant (figure $3(d)$ ) (for more details, see the supplementary data and figure S2). Significantly, these traces do not exhibit the substantial time-delayed displacements between both mitochondrial ends that we see in the experiment.

Statistical analysis of the standard deviations of the mitochondrial length $d$ and width $d_{y}$ taken from the experiment $\left(\sigma_{d}=48.6 \mathrm{~nm}\right.$ and $\left.\sigma_{d_{y}}=34.4 \mathrm{~nm}\right)$ and the simulation $\left(\sigma_{d}=33.5 \mathrm{~nm}\right.$ and $\left.\sigma_{d_{y}}=30.1 \mathrm{~nm}\right)$ reveals a significantly increased standard deviation for the mitochondrial length $(P<0.009$, equation (1)), whereas the standard deviations for the experimental and simulated mitochondrial width were statistically indistinguishable $(P<0.36)$ (see the supplementary data available from stacks.iop.org/PhysBio/3/45 for more details).

Next, we examined the mitochondrial size changes observed, using the zero-crossing analysis (Materials and methods). The expected mean number of zero crossings (size transitions) for a total of 100 images is 49.5. The experiment results in $37\left(P^{-}<0.006\right.$, equation (2)) and $45\left(P^{-}<0.18\right)$ zero crossings for the length and width, respectively, demonstrating a significantly decreased number of zero crossings along the direction of transport (see also supplementary figure $\mathrm{S} 1(c))$. In contrast, both zerocrossing numbers gained from the simulation are close to the expectation (52, $P^{+}<0.31$, equation (2)). This strongly suggests systematic length changes of the measured mitochondrion, a result in agreement with the observed timedelayed movements of the mitochondrial ends.

Figure 4 shows an example of an apparently immobile mitochondrion together with a mobile mitochondrion (a detailed analysis of an oscillating forward and backward moving mitochondrion is provided in the supplementary data, see figure S4). Figure 4(a) depicts the intensity distributions together with the CLSM micrograph of both mitochondria. Shown is the first image of 90 sequential images. The trajectories of the object points $p_{0}$ to $p_{4}$ (see the inset of figure $4(c)$ ) are shown in figure $4(b)$. The mitochondrion on the right-hand side moves toward the center of the image in the retrograde direction while the mitochondrion on the lefthand side appears to be immobile. The displacement traces of the front and rear ends of both mitochondria after applying a three-point moving window are shown in figures $4(c)$ and (d) (the original data traces are shown in supplementary figures $\mathrm{S} 3(a)$ and $(b)$ ). Similar to the example shown in figure 3(a), transport of the mobile mitochondrion appears to be accomplished in a linear fashion with sections of different mean velocities during continuous unidirectional movement (inset, figure $4(e)$ ). Both mitochondria seem to undergo persistent length and width changes (figures $4(c)$ and $(d)$ ). Here, zero-crossing analysis results in $32\left(P^{-}<0.004\right)$ and 


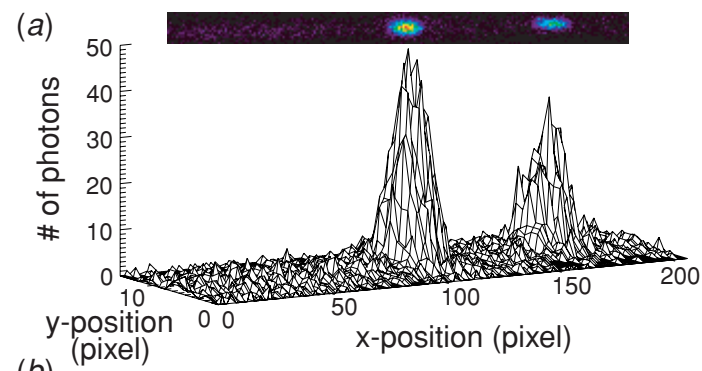

$(d)$
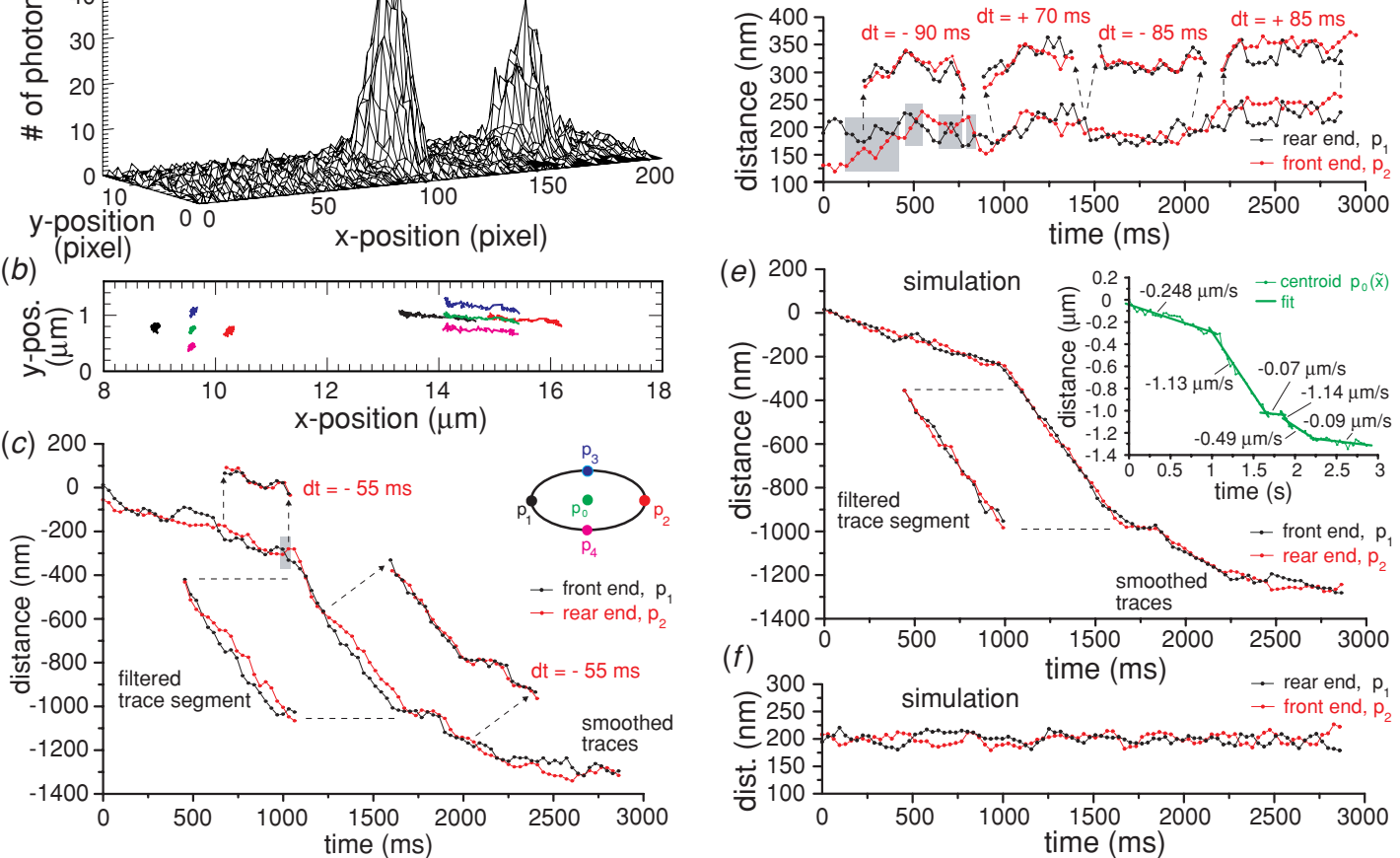

Figure 4. Simultaneous measurement of an apparently immobile and mobile dendritic mitochondrion. (a) Intensity distributions of both mitochondria in the first of 90 sequential images (the corresponding CLSM micrograph is shown in the inset). CLSM image size: $208 \times 16$ pixel. Image sampling interval: $32.16 \mathrm{~ms}$. Line scanning time: $2.01 \mathrm{~ms}$. Pixel dwell time: $5.15 \mu$ s. Pixel size: $88 \mathrm{~nm}$ (for parameters characterizing the object intensities, see supplementary table S2). (b) Trajectories of the object points $p_{0}$ to $p_{4}$ of both mitochondria (see inserted schematic in $(c)$ of this figure). (c) Displacement traces (with respect to an axis parallel to the major mitochondrial symmetry axis) of the front and rear ends of the mobile mitochondrion after applying a three-point moving window to the original traces shown in supplementary figure S3(a) (mean transport direction: $\varphi=-0.0398 \pm 0.0215$ ). The inserted trace segment on the left-hand side depicts a characteristic part of the mitochondrial displacement traces after applying a $0.01 \mathrm{~Hz}$ low-pass filter to the original traces (the dashed lines mark the corresponding part of the smoothed traces). The inserted trace segments (see dashed arrows) were superimposed by shifting the corresponding parts of the front end trace by $-55 \mathrm{~ms}$ in time. (d) Smoothed displacement traces of the apparently immobile mitochondrion (the original traces are shown in supplementary figure S3(b)) (mean object orientation: $\varphi=-0.0486 \pm 0.0317$ ). The insets show superimposed trace segments of the mitochondrial front and rear ends ( $\mathrm{d} t$ indicates the corresponding time delays). (e) Smoothed displacement traces of the front and rear ends of the simulated mobile mitochondrion (see supplementary data for a detailed description of the underlying simulation). The inserted trace segment depicts a low-pass-filtered part of the original traces shown in supplementary figure S3 $(c)$. The graph above the smoothed traces shows the original displacement trace of the centroid $\left(p_{0}\right)$ (with respect to an axis parallel to the major mitochondrial symmetry axis) of the measured mobile mitochondrion. The superimposed straight lines depict the result of the fits to the various trace sections. $(f)$ Smoothed displacement traces of the front and rear ends of the simulated immobile mitochondrion (see supplementary data and figure $\mathrm{S} 3(d)$ for more information).

$39\left(P^{-}<0.12\right)$ zero crossings for the length and width of the mobile mitochondrion (the expected mean number of zero crossings for 90 images is 44.5$)$ and in $41\left(P^{-}<0.23\right)$ and $43\left(P^{-}<0.38\right)$ zero crossings for the length and width of the immobile mitochondrion, respectively. The persistence of size changes is thus statistically validated only for the length changes of the mobile mitochondrion.

The traces of the mobile mitochondrion again reveal similar time-delayed displacements of both ends (figure 4(c)). To help visualize characteristic features present in the original data traces (supplementary figure $\mathrm{S} 3(a)$ ), we applied a $0.01 \mathrm{~Hz}$ low-pass filter and inserted a segment of the resulting traces (inset, figure 4(c)). Apparently, the front end dictates movement and the rear end lags behind, a feature reminiscent of an inchworm-like motility mechanism. The simulated mitochondrion, on the other hand, does not exhibit such a characteristic feature (see the filtered trace segment in figure $4(e)$ ). Within this segment, the measured non-averaged traces do not cross each other for 14 sampling intervals (supplementary figure S3(a)). The probability for such an event is 0.005 (see the supplementary data available from stacks.iop.org/PhysBio/3/45). This result clearly indicates systematic mitochondrial length changes over the entire duration of the depicted trace segment (figure 4(c)).

Mitochondrial length changes and the similarity of the time-delayed displacements of both mitochondrial ends appear to be a typical feature of mitochondria transport: For a proofof-principle, we took 25 measurements of actively transported mitochondria (all from dendrites of different neurons) from which eight measurements met the described criteria for reliable FPT analysis (see section 'finite-particle tracking (FPT) analysis') and provided a sufficient SNR (the remainders were discarded due to extensive photobleaching and/or the 
release of the accumulated dye during image acquisition and/or a poor mitochondrial uptake of the fluorescent dye). All eight measurements revealed statistical significant mitochondrial length fluctuations (judged by SD analysis). Although all traces also showed segments of apparent timedelayed similar displacements (see, e.g., supplementary figure S4), only four experiments provided a sufficiently high tracking precision to allow their validation via comparison with simulated traces (see figures 3 and 4 and supplementary figure S5), demonstrating the need for a significantly increased SNR using a different staining strategy (see Discussion).

The inserted superimposed trace segments in figures 4(c) and $(d)$ demonstrate once again the similarity of the movements of both ends $(\mathrm{d} t$ indicates the approximate time delay). Such substantial time-delayed movements are absent in simulated traces (figures $4(e)$ and $(f)$, see also supplementary figure S3). Importantly, the rear end of the mobile mitochondrion (figure 4(c)) always lags behind the front end, whereas the front and rear ends of the immobile mitochondrion (figure $4(d)$ ) apparently switch leading and lagging roles (alternating negative and positive time delays of $\sim 80 \mathrm{~ms})$. Moreover, the front and rear ends of the immobile mitochondrion seem to undergo substantial simultaneously opposing displacements during the first $800 \mathrm{~ms}$ of data acquisition (see, gray-shaded rectangles in figure $4(d)$ ), suggestive for a tug-of-war between both ends. This interpretation is supported by the almost identical timedelayed displacements of both ends (see inserted superimposed trace segments on the left-hand side of figure $4(d)$ ), by the comparison with the simulated traces (figure $4(f)$ ) and the statistically validated length changes of this mitochondrion (see above). The apparently immobile mitochondrion does, thus, appear to exhibit substantial size changes presumably due to competitive activity at both mitochondrial ends. Collectively, our data clearly show that mitochondria undergo characteristic size changes during movement.

\section{Discussion}

We have applied a recently developed FPT algorithm [24] and analyzed mitochondria transport in dendrites of cultured neurons. Quantitative analysis of the data obtained revealed transport-induced submicroscopic-size changes of mitochondria. Front and rear ends of mitochondria can undergo similar characteristic movements with substantial time delay between the displacements of both ends, an observation reminiscent of inchworm-like locomotion. Furthermore, we have demonstrated that both mitochondrial ends can play distinctive roles during transport-while one of the ends leads, the other lags behind. Both the observed alternating leading roles and the detected simultaneously opposing displacements of the mitochondrial ends could suggest an occasional tug-of-war between both ends.

To quantitatively analyze shape and size changes of mitochondria, we have explicitly taken into account the noise-limited localization precision of object borders and simulated fluorescence images of mitochondria by adopting the object parameters and noise characteristics of a given experiment. By performing statistical and zero-crossing analysis of experimental and simulated data, we have shown that processively transported mitochondria can undergo persistent submicroscopic-length changes. As the direction of length changes coincides with the direction of transport, our investigation suggests the length changes to be transport related. Mitochondrial length changes were in part brought about by non-simultaneous displacements of both ends. As the time-delayed movements of either cargo ends had the same mean velocity (identical shape and curvature of the smoothed trace segments, see figures $3(b)$ and $4(c)$ ), we propose these movements to be motor-driven rather than being caused by elastic forces that could potentially lead to some type of 'oscillatory' or 'jumping' motion of the lagging end.

Several processes could potentially cause shape and size changes of the cargo (motion blur caused by the line-scanning process of the laser scanning microscope can be neglected, see the supplementary data available from stacks.iop.org/PhysBio/3/45). (i) unsynchronized activity of motor proteins attached to the front and rear ends of the cargo could potentially cause size changes, (ii) obstacles in the relatively crowded intracellular environment could hamper simultaneous movement of both cargo ends and (iii) switching between the end of one microtubule to the beginning of the next might interfere with simultaneous displacements of both cargo ends. However, those processes are unlikely to account for the observed time-delayed similar movements of both cargo ends (e.g., direction reversals or movement with a certain speed followed by a characteristic pause, see figures 3(b) and $4(c)$ and $(d))$. The finding that the time-delayed movements of the leading and trailing mitochondrial ends were almost identical (see above) and that they occurred at spatially different positions in the dendrite (more than $1 \mu \mathrm{m}$ apart in the presented examples, see figure $2(d)$ ) rather suggests that the (motor) activity at one end is somehow communicated to the other end. What signal could spread along the length of a mitochondrion in about 50-150 ms (see figures 3 and 4)? Signal pathways could potentially involve forces exerted along the cargo membrane. Although forces that activate/deactivate or modulate motor function via strain-sensitive motor-cofactor proteins cannot be yet ruled out, they are expected to take effect in a time much faster than the measured time delay of tens of milliseconds [28]. In a different scenario, forces could act directly on motor proteins and cause motor stalling or even make them moving backward [29] (for a detailed discussion on a tug-of-war between motor proteins, see the supplementary data available from stacks.iop.org/PhysBio/3/45) though such a mechanism (when continuously utilized) seems to be too inefficient from the energetic point of view and not supported by the current state of research [8]. A principally different pathway, on the other hand, could involve waves of signal molecules that propagate along the cargo membrane. Given a mitochondrial length of about $1.3 \mu \mathrm{m}$ (see examples in figures 2 and 4), this signal must travel with a speed in the range of 9-26 $\mu \mathrm{m} \mathrm{s}^{-1}$. Thus, waves of second messengers such as $\mathrm{Ca}^{2+}$ or cyclic adenosine $3^{\prime}, 5^{\prime}$-monophosphate (cAMP) would be good candidates as these waves are known to 
travel over long distances across the cell with a speed in the range of $8-100 \mu \mathrm{m} \mathrm{s}^{-1}$ [30] and are likely involved in the regulation of bidirectional organelle transport via $\left[\mathrm{Ca}^{2+}\right]$ and [cAMP]-dependent phosphorylation/dephosphorylation of motor proteins [21-23, 31, 32]. In particular, the speed of $\mathrm{Ca}^{2+}$ waves in astrocytes and neurites of PC12 cells and neurons has been determined to be in the range of 5-30 $\mu \mathrm{m} \mathrm{s}^{-1}$ [33, 34], 17-30 $\mu \mathrm{m} \mathrm{s}^{-1}$ [35] and $10 \mu \mathrm{m} \mathrm{s}^{-1}$ [36], respectively, in good agreement with the anticipated velocity range. Besides $\left[\mathrm{Ca}^{2+}\right]$-sensitive phosphorylation/dephosphorylation of motor proteins, $\left[\mathrm{Ca}^{2+}\right]$-dependent motor regulation by calmodulin might play a role $[37,38]$. Taken together, the detected time-delayed movements of both mitochondrial ends might provide new hints for the mechanism underlying bidirectional organelle transport.

The observed simultaneously opposing displacements of the leading and trailing ends of mitochondria could in particular indicate an occasional tug-of-war between oppositepolarity motor proteins or motor proteins of the same directionality but moving along anti-parallel microtubules since microtubules are of mixed orientation in proximal dendrites [39, 40]. Whereas an occasional tug-ofwar between motors of the same type that carries a mitochondrion through a network of anti-parallel microtubules is not an unexpected scenario [7], competitive activity of opposite-polarity motors in processes with uniform aligned microtubules is controversial [8]. In fact, a recent highprecision single-particle tracking (SPT) study on actively transported GFP-tagged peroxisomes in S2 cells [28] strongly argues against a tug-of-war scenario in such processes. However, as is typical for studies that provide indirect evidence for a proposed mechanism, the same data can be interpreted in favor of a model partially based on a tug-ofwar between motor molecules (see the supplementary data available from stacks.iop.org/PhysBio/3/45), demonstrating the need for direct evidence for the underlying mechanism of bidirectional organelle transport.

To prove that our observations are of a general nature and not restricted to mitochondria transport in proximal dendrites, transport in distal dendrites [39, 40] or axonal transport has to be analyzed. However, a thorough study of the reported processes in dendrites and axons that does not require extensive computer simulations awaits both an improved tracking precision and an increased time resolution. The low excitation intensity and small pixel dwell time (to prevent photodamage of mitochondria), the photodetector deadtime and the use of an exogenous dye for the staining of mitochondria prevented us from achieving tracking precisions in the lower nanometer range [24]. Despite the modest tracking precision, we were able to gain statistically validated information about mitochondrial size changes using computer simulations.

\section{Conclusion and outlook}

Our study unequivocally revealed transport-induced submicroscopic-size changes of mitochondria and thus demonstrated the potential impact of shape and size analysis of actively transported finite objects. In combination with an improved time resolution, an increased tracking precision and the use of RNAi analysis (RNAi has not yet been undertaken in neurons from Xenopus laevis; therefore, other neuronal cell systems such as cultured rat sympathetic neurons could be tried out, see [41]) the provided biophysical framework will prove to be an excellent tool for studying the mechanism of bidirectional organelle transport in living cells. Deciphering the source of the observed simultaneously opposing displacements and the time-delayed similar movements of both mitochondrial ends and the potential simultaneous detection of opposing motor steps could have profound implications for the mechanism underlying bidirectional organelle transport.

\section{Acknowledgments}

The authors wish to thank N O Petersen for stimulating and invaluable discussions and C Wald and B Beyerstedt for help with statistical analysis. This work has been supported by DFG Research Center of Molecular Physiology of the Brain (DS), DFG grant GE 1609/1 (AG), and BCCN grant 01GQ0432 (DS).

\section{Glossary}

Tug-of-war. Motor proteins pulling against each other.

Zero crossings. Commonly defined as the number of times a signal crosses the zero level, with either a positive or negative slope. Here it is defined as the number of times a mitochondrion changes its length (or width, respectively) between images from a value larger than the measured mean length (or width) to a value smaller than the measured mean length (or width) or vice versa.

Finite-particle tracking (FPT). Localization and tracking of a finite-sized fluorescent object in subsequent images. The size and shape of the three-dimensional object are taken into account by the FPT algorithm, in contrast to a SPT algorithm in which the object (commonly a fluorescent molecule) is assumed to be a point object.

Anterograde transport. Movement toward the synapse. Retrograte transport. Movement toward the cell body.

\section{References}

[1] Hollenbeck P J 1996 The pattern and mechanism of mitochondrial transport in axons Front. Biosci. 1 d91-120

[2] Brady S T 1991 Molecular motors in the nervous system Neuron 7 521-33

[3] Hirokawa N 1998 Kinesin and dynein superfamily proteins and the mechanism of organelle transport Science 279 519-26

[4] Vale R D 2003 The molecular motor toolbox for intracellular transport Cell 112 467-80

[5] Hirokawa N and Takemura R 2005 Molecular motors and mechanisms of directional transport in neurons Nat. Rev. Neurosci. 6 201-14

[6] Schliwa M 2003 Molecular Motors (Germany: VCH-Wiley)

[7] Welte M A 2004 Bidirectional transport along microtubules Curr. Biol. 14 R525-37 
[8] Gross S P 2004 Hither and Yon: a review of bi-directional microtubule-based transport Phys. Biol. 1 R1-11

[9] Hirokawa N, Sato-Yoshitake R, Yoshida T and Kawashima T 1990 Brain dynein (MAP1C) localizes on both anterogradely and retrogradely transported membranous organelles in vivo J. Cell Biol. 111 1027-37

[10] Ma S and Chisholm R L 2002 Cytoplasmic dynein-associated structures move bidirectionally in vivo J. Cell Sci. 115 1453-60

[11] Gross S P, Tuma M C, Deacon S W, Serpinskaya A S, Reilein A R and Gelfand V I 2002 Interactions and regulation of molecular motors in Xenopus Melanophores J. Cell Biol. 156 855-65

[12] Gross S P, Guo Y, Martinez J E and Welte M A 2003 A determinant for directionality of organelle transport in Drosophila embryos Curr. Biol. 13 1660-8

[13] De Vos K J, Sable J, Miller K E and Sheetz M P 2003 Expression of phosphatidylinositol $(4,5)$ bisphosphatespecific pleckstrin homology domains alters direction but not the level of axonal transport of mitochondria Mol. Biol. Cell 14 3636-49

[14] Ligon L A, Tokito M, Finklestein J M, Grossman F E and Holzbaur E L 2004 A direct interaction between cytoplasmic dynein and kinesin I may coordinate motor activity J. Biol. Chem. 279 19201-08

[15] Gross S P, Welte M A, Block S M and Wieschaus E F 2002 Coordination of opposite-polarity microtubule motors J. Cell Biol. 156 715-24

[16] Waterman-Storer C M, Karki S B, Kuznetsov S A, Tabb J S, Weiss D G, Langford G M and Holzbaur E L 1997 The interaction between cytoplasmic dynein and dynactin is required for fast axonal transport Proc. Natl Acad. Sci. USA 94 12180-5

[17] Martin M, Iyadurai S J, Gassman A, Gindhart J G Jr, Hays T S and Saxton W M 1999 Cytoplasmic dynein, the dynactin complex, and kinesin are interdependent and essential for fast axonal transport Mol. Biol. Cell $103717-28$

[18] Valetti C, Wetzel D M, Schrader M, Hasbani M J, Gill S R, Kreis T E and Schroer T A 1999 Role of dynactin in endocytic traffic: effects of dynamitin overexpression and colocalization with CLIP-170 Mol. Biol. Cell 10 4107-20

[19] Deacon S W, Serpinskaya A S, Vaughan P S, Lopez Fanarraga M, Vernos I, Vaughan K T and Gelfand V I 2003 Dynactin is required for bidirectional organelle transport J. Cell Biol. 160 297-301

[20] Welte M A, Gross S P, Postner M, Block S M and Wieschaus E F 1998 Developmental regulation of vesicle transport in Drosophila embryos: forces and kinetics Cell 92 547-57

[21] Sato-Yoshitake R, Yorifuji H, Inagaki M and Hirokawa N 1992 The phosphorylation of kinesin regulates its binding to synaptic vesicles J. Biol. Chem. 267 23930-6

[22] Dillman J F III and Pfister K K 1994 Differential phosphorylation in vivo of cytoplasmic dynein associated with anterogradely moving organelles J. Cell Biol. 127 1671-81

[23] Lee K D and Hollenbeck P J 1995 Phosphorylation of kinesin in vivo correlates with organelle association and neurite outgrowth J. Biol. Chem. 270 5600-05
[24] Gennerich A and Schild D 2005 Sizing-up finite fluorescent particles with nanometer-scale precision by convolution and correlation image analysis Eur. Biophys. J. 34 181-99

[25] Bischofberger J and Schild D 1995 Different spatial patterns of $\left[\mathrm{Ca}^{2+}\right]$ increase caused by $\mathrm{N}$ - and L-type $\mathrm{Ca}^{2+}$ channel activation in frog olfactory bulb neurons $J$. Physiol. 487 $305-17$

[26] Gennerich A and Schild D 2000 Fluorescence correlation spectroscopy in small cytosolic compartments depends critically on the diffusion model used Biophys. J. 79 3294-306

[27] Papoulis A and Pillai S U 2002 Probability, Random Variables, and Stochastic Processes 4th edn (New York: McGraw-Hill)

[28] Kural C, Kim H, Syed S, Goshima G, Gelfand V I and Selvin P R 2005 Kinesin and dynein move a peroxisome in vivo: a tug-of-war or coordinated movement? Science 308 1469-72

[29] Carter N J and Cross R A 2005 Mechanics of the kinesin step Nature 435 308-12

[30] Meyer T 1991 Cell signaling by second messenger waves Cell 64 675-8

[31] Donelan M J et al $2002 \mathrm{Ca}^{2+}$-dependent dephosphorylation of kinesin heavy chain on $\beta$-granules in pancreatic $\beta$-cells J. Biol. Chem. 277 24232-42

[32] Hollenbeck P J 1993 Phosphorylation of neuronal kinesin heavy and light chains in vivo J. Neurochem. $602265-75$

[33] Charles A C and Giaume C 2002 The Tripartite Synapse ed A Volterra, P J Magistretti and P G Haydon (New York: Oxford University Press) pp 110-26

[34] Bernardinelli Y, Magistretti P J and Chatton J Y 2004 Astrocytes generate $\mathrm{Na}^{+}$-mediated metabolic waves Proc. Natl Acad. Sci. USA 101 14937-42

[35] Lorenzon P, Zacchetti D, Codazzi F, Fumagalli G, Meldolesi J and Grohovaz F $1995 \mathrm{Ca}^{2+}$ waves in PC12 neurites: a bidirectional, receptor-oriented form of $\mathrm{Ca}^{2+}$ signaling J. Cell Biol. 129 797-804

[36] Ennes H S, Young S H, Raybould H E and Mayer E A 1997 Intercellular communication between dorsal root ganglion cells and colonic smooth muscle cells in vitro NeuroReport $8733-7$

[37] Hisanaga S and Sakai H 1983 Cytoplasmic dynein of the sea urchin egg: II. Purification, characterization and interactions with microtubules and Ca-calmodulin J. Biochem. (Tokyo) 93 87-98

[38] Hisanaga S and Pratt M M 1984 Calmodulin interaction with cytoplasmic and flagellar dynein: calcium-dependent binding and stimulation of adenosinetriphosphatase activity Biochemistry 23 3032-7

[39] Burton P R 1988 Dendrites of mitral cell neurons contain microtubules of opposite polarity Brain Res. 473 107-15

[40] Baas P W, Deitch J S, Black M M and Banker G A 1988 Polarity orientation of microtubules in hippocampal neurons: uniformity in the axon and nonuniformity in the dendrite Proc. Natl Acad. Sci. USA 85 8335-9

[41] He Y, Francis F, Myers K A, Yu W, Black M M and Baas P W 2005 Role of cytoplasmic dynein in the axonal transport of microtubules and neurofilaments $J$. Cell Biol. 168 697-703 